\title{
Synthesis and Characterization of CdS Nanoparticles Grown in a Polymer Solution using in-situ Chemical Reduction Technique
}

\author{
Shujahadeen B. Aziz ${ }^{1}$, Mariwan A. Rasheed ${ }^{2}$, Salah R. Saeed ${ }^{3}$, Omed Gh. Abdullah ${ }^{1, *}$ \\ ${ }^{1}$ Advanced Materials Research Lab., Department of Physics, College of Science, University of \\ Sulaimani, 46001, Kurdistan Region, Iraq. \\ ${ }^{2}$ Development Centre for Research and Training (DCRT), University of Human Development, \\ Kurdistan Region, Iraq. \\ ${ }^{3}$ Charmo Research Center, Charmo University, Peshawa Street, Chamchamal, 46023, Sulaimani, \\ Kurdistan Region, Iraq. \\ *E-mail: omed.abdullah@ univsul.edu.iq
}

doi: $10.20964 / 2017.04 .10$

Received: 3 January 2017 / Accepted: 12 February 2017 / Published: 12 March 2017

Cadmium sulfide (CdS) nanoparticles embedded in poly(vinyl alcohol) (PVA) in the form of films with different $\mathrm{CdS}$ content have been prepared successfully using in-situ chemical reduction technique and casting of aqueous solutions. The nano-CdS characterised using UV-Vis spectroscopy which shows an absorption shoulder around $500 \mathrm{~nm}$ confirming the formation of CdS nanoparticles. Moreover, the linear increase in absorbance with increasing CdS concentration revealed that the $\mathrm{CdS}$ nanoparticles are homogeneously distributed in the host PVA polymer matrix. To detect structural changes, XRD and FTIR spectra of pure and CdS doped PVA films have been analysed. FTIR analysis evidently shows the interaction between CdS nanoparticles with the hydroxyl groups of PVA. It is also found that the ac conductivity of doped PVA samples increases with the increase of CdS content as well as frequency. The calculated values of frequency exponent $(s)$ suggest that the electrical conduction is followed by the correlated barrier hopping $(\mathrm{CBH})$ model. The temperature dependence of ac conductivity also indicated that the minimum activation energy associated with the maximum conductivity composition. The experimental results reveal that the optical and electrical behaviour of PVA can be improved significantly by the addition of a small amount of CdS nanoparticles. This enhanced properties of PVA/CdS nanocomposite proposed to be used in optoelectronic devices.

Keywords: polymer nanocomposites; CdS nanoparticles; conduction mechanism; ac conductivity; activation energy.

$\underline{\text { FULL TEXT }}$ 
(C) 2017 The Authors. Published by ESG (www.electrochemsci.org). This article is an open access article distributed under the terms and conditions of the Creative Commons Attribution license (http://creativecommons.org/licenses/by/4.0/). 